\title{
Sarcomatoid Carcinoma of the Urinary Bladder: A Case Report and Review of the Literature
}

\author{
Jose Emilio Hernandez Sanchez ${ }^{\mathrm{a}, \mathrm{c}}$, Yuri Rodrigues Figueira ${ }^{\mathrm{b}}$, Raquel Tur Gonzalez ${ }^{\mathrm{b}}$, \\ Rocio Martin Lopez ${ }^{b}$, Luis Miguel Gomez Tejeda ${ }^{a}$
}

\begin{abstract}
A case of sarcomatoid carcinoma of the urinary bladder is reported. An 81-year-old man with sarcomatoid carcinoma underwent radical cistectomy was alive without recurrence after six months. The characteristics of the neoplasm are reported and discussed.
\end{abstract}

Keywords: Sarcomatoid carcinoma; Bladder cancer; Non-urothelial tumor

\section{Introduction}

Transitional cell carcinoma is the most prevalent bladder neoplasm. Non-urothelial bladder tumors frequently present a challenge and may not be encountered within a lifetime of practicing urology [1]. Sarcomatoid carcinoma is an uncommon malignancy with distinctive therapeutic and prognostic implications. In the urinary bladder, it represents only $0.1 \%$ to $0.3 \%$ of carcinomas [2].

\section{Case Report}

An 81-year-old man with macrohematuria was referred in June 2013 to our hospital. CT revealed a large mass occupying almost the entire bladder and no metastatic lesions (Fig. 1A). Transurethral bladder tumor resection was performed. The pathological diagnosis was infiltrative sarcomatoid carcinoma. Due to the inability to control tumor by reiterative transurethral resection a radical cistectomy with lymph node

\footnotetext{
Manuscript accepted for publication December 13, 2013

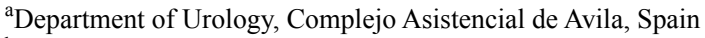

${ }^{\mathrm{b}}$ Department of Pathology, Complejo Asistencial de Avila, Spain

${ }^{c}$ Corresponding author: Hernandez Sanchez JE, Department of Urology

Complejo Asistencial de Avila. Avda. Juan Carlos I S/N; 05071-Avila,

Spain. Email: dr_emilio_hernandez@hotmail.com

doi: http://dx.doi.org/10.14740/jmc1657w
}

dissection and ureterostomy were performed to control hematuria. The postoperative course was uneventful.

Grossly it was a large and polipoid tumor, fleshy color, fulfilling the whole bladder lumen, with infiltration through the muscularis wall and reaching the adventitia. Microscopic examination revealed a high grade spindle cell tumor without heterologous differentiation; with vimentin, epithelial membrane antigen (EMA) and keratin (AE1/AE3) positivity. Antibodies for specific mesenchymal differentiation were negative.

Medical Oncology Service misestimated adjuvant chemotherapy and radiotherapy. Six months after surgery the patient is alive without tumor recurrence and good life quality.

\section{Discussion}

The term sarcomatoid variant of urothelial carcinoma should be used for all biphasic malignant neoplasms exhibiting evidence of epithelial and mesenchymal differentiation, with the presence or absence of heterologous elements [3].

There is disagreement regarding the nomenclature and histogenesis of these tumors. Various terms have been used

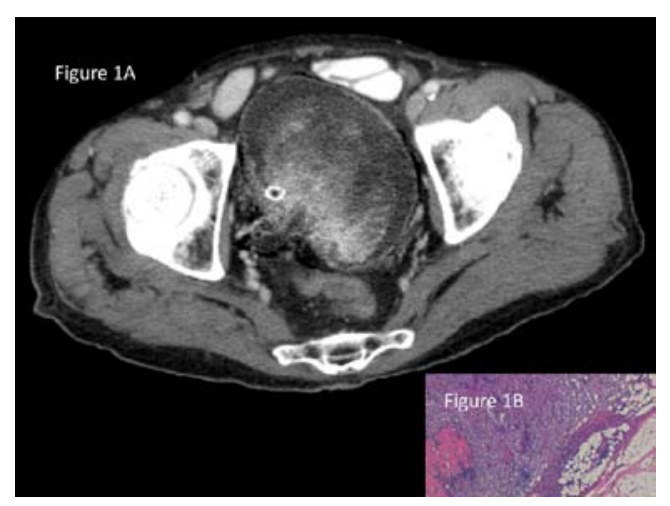

Figure 1. A). CT revealed a large mass occupying almost the entire bladder at an advanced stage disease (T2 or higher) and no metastatic lesions. B). Sarcomatoid carcinoma mostly composed of undifferentiated malignant spindle cells. Tumor infiltrates through adventitial adipose tissue. 


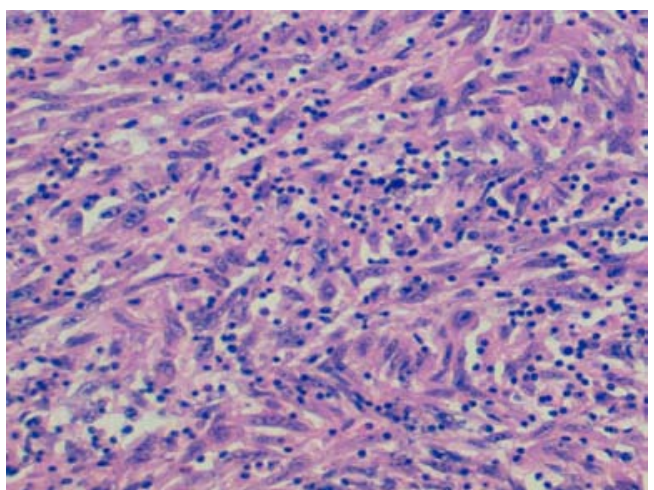

Figure 2. Sarcomatoid carcinoma composed entirely of sarcomatous appearing cells.

for these neoplasms, including carcinosarcoma. In some series, both are included as sarcomatoid carcinoma. In others, they are regarded as separate entities $[2,3]$. Sarcomatoid carcinoma is the recommended terminology by the WHO for these lesions and carcinosarcoma should be used as a synonym rather than recognized as a distinct entity.

It most commonly affects white elderly male patients in their seventh decade of life. The risk factors are the same for usual urothelial carcinoma. Radiotherapy or cyclophosphamide has been reported as specific risk factors [1-3].
Symptoms are similar to those with typical urothelial carcinoma. The most frequent presenting features are hematuria, dysuria, nocturia, acute urinary retention, and lower abdominal pain [1-3].

Although sarcomatoid carcinoma can arise in any portion of the urothelium, it is rare in extravesical sites. It is most frequently found on the lateral walls of the bladder. Cystoscopy shows broad-based and often polypoid neoplasm rapidly growing into the lumen of the bladder. $\mathrm{CT}$ and MRI frequently presents at an advanced stage disease (T3 or higher) [3-6].

On macroscopic examination, the tumors are typically large, either polypoid or pedunculated. Edema, hemorrhage, necrosis and cysts are often evident. Grossly evident infiltration of extravesical soft tissues is common [3].

Histology reveals a tumor showing both a malignant spindle cell component and a malignant epithelial component, although the latter may only be identifiable immunohistochemically. The spindle cell component is usually high grade with nondescript architecture (often resembling malignant fibrous histiocytoma) comprising $>50 \%$ of the tumor in most cases (Fig. 1B, 2). It may merge with foci of overlying CIS or with invasive urothelial carcinoma, squamous cell carcinoma, adenocarcinoma, or small cell carcinoma. Hetelogous differentiation in the form of rhabdomyosarcomatous, osteosarcomatous and chondrosarcomatous may be present; but it is not necessary to perform the diagnosis and

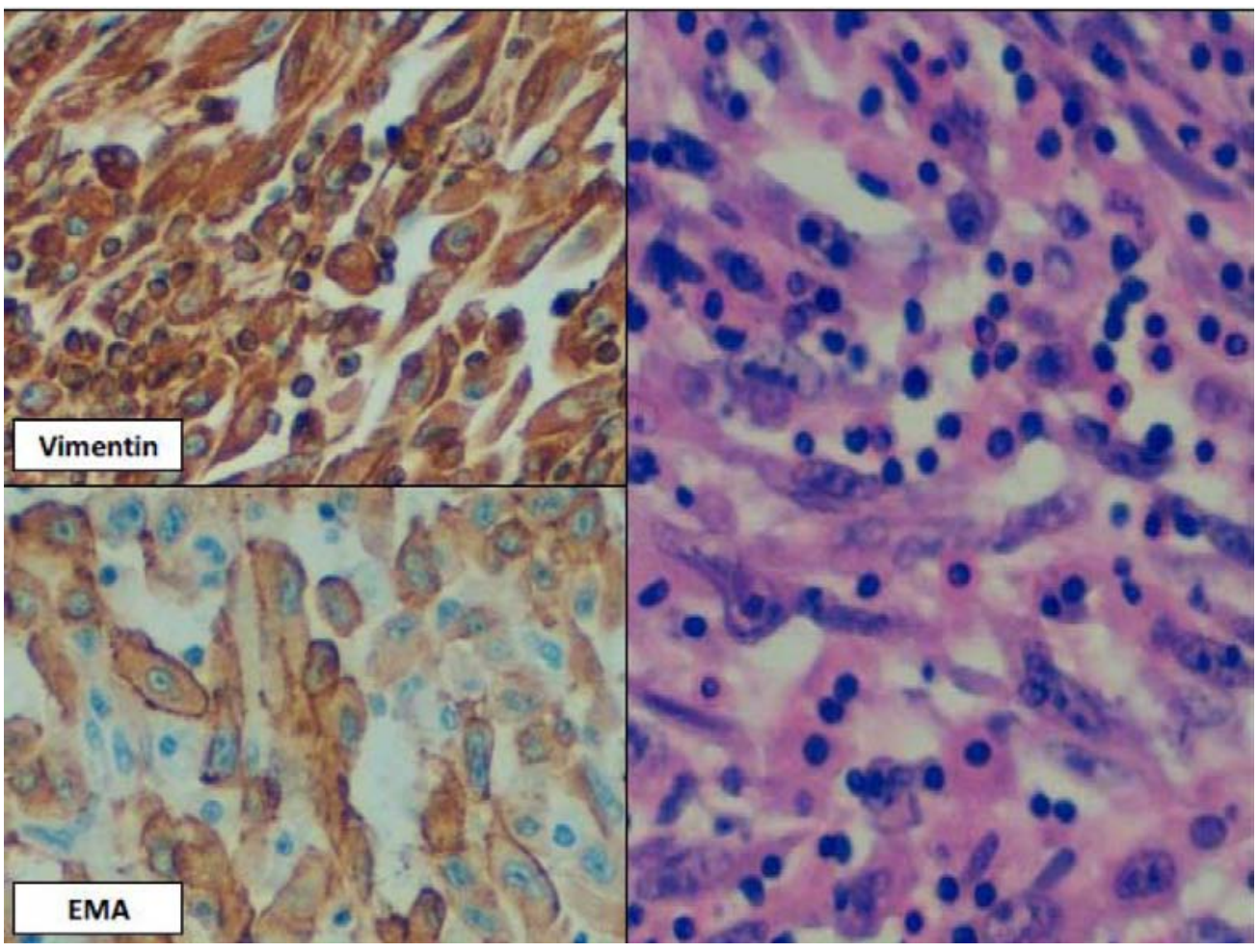

Figure 3. Higher magnification of Fig. 2 with vimentin and EMA positivity documenting epithelial differentiation. 
Sarcomatoid Carcinoma

J Med Cases. 2014;5(2): I I6-II9

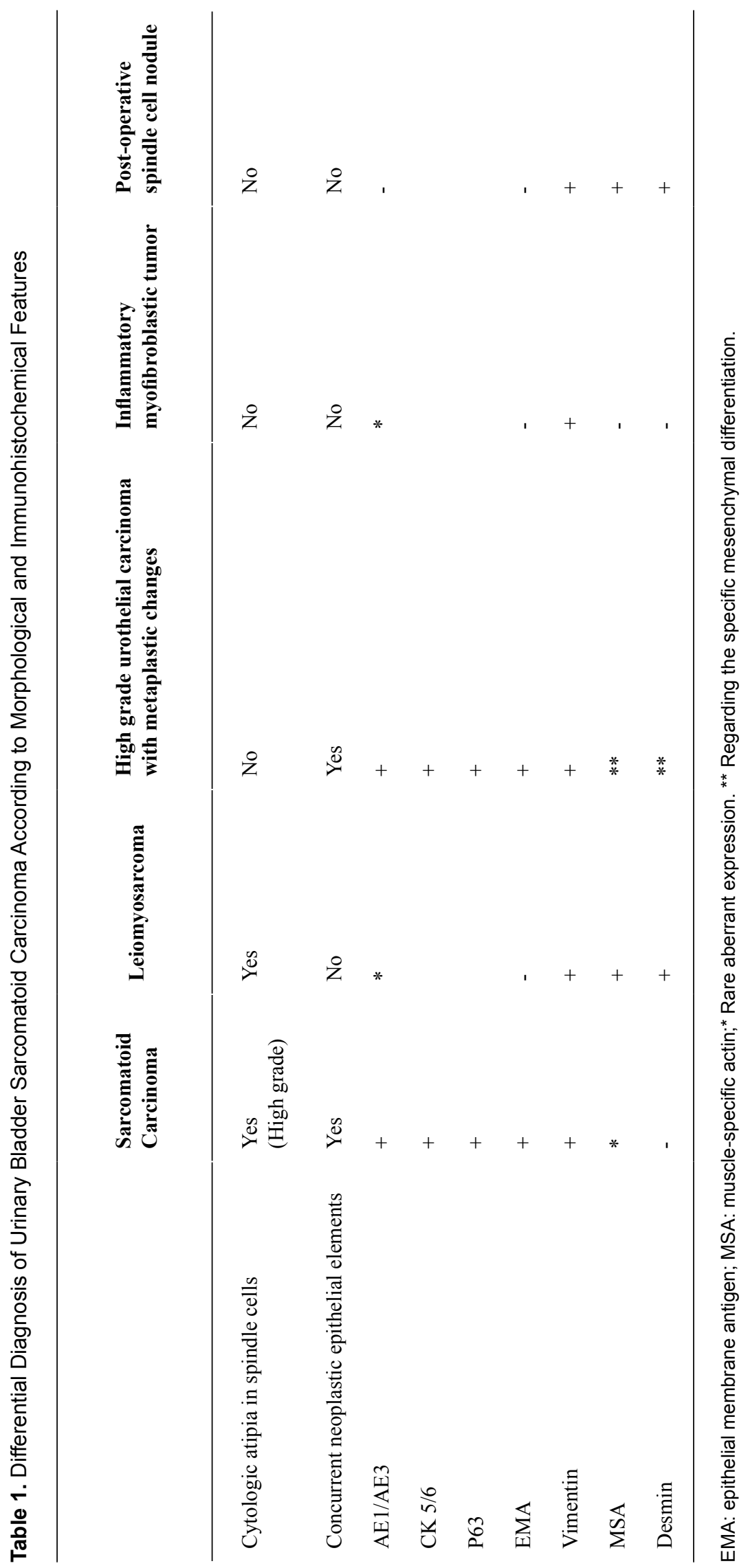

118

Articles (C) The authors | Journal compilation @ J Med Cases and Elmer Press Inc ${ }^{\mathrm{TM}}$ | www.journalmc.org 
to date has no prognostic significance. Osteosarcoma is the most common heterologous element. More than one type of heterologous differentiation may be present $[2,3,6]$.

By immunohistochemistry, the carcinomatous component is universally positive for epithelial markers EMA and pan-keratin, and positive for vimentin in approximately $80 \%$ to $90 \%$ of tumors (Fig. 3 ). In contrast, the sarcomatous component reacts with vimentin only, while variably stains for keratin AE1/AE3, keratin CAM 5.2, and EMA, but usually shows positivity for at least one marker. It may express specific markers for specific mesenchymal differentiation if present $[2,3]$

Sarcomatoid carcinoma seems to represent the final common and most aggressive pathway of human tumor dedifferentiation, including bladder cancer. Two theories have been proposed to explain the origin of these biphasic tumors. Some believe that it represents a collision tumor of two independent monoclonal neoplasms occurring simultaneously, whereas others suggest that has a common clonal origin with divergent differentiation into its elements. Recent molecular studies support the latter theory [2, 3].

Differential diagnosis includes a wide variety of entities, including benign and malignant proliferations (Table 1). In view of the rarity of primary bladder sarcoma, any malignant spindle cell tumor in the urinary bladder of an adult is considered sarcomatoid carcinoma until proven otherwise $[2,3]$.

Most patients with carcinosarcoma of the urinary bladder had high-histological grade and advanced stage disease at the time of presentation. It shows an unfavorable prognosis worse than high-grade urothelial carcinoma. Most authors have reported poor outcomes that relates to an aggressive biological behavior as well as to an often times advanced stage at the time of diagnosis. Tumor stage has been identified as an independent factor associated with cancer specific survival. The mean survival of patients is $1-2$ years despite aggressive surgical management. Patients with regional and distant spread of disease have a 2 -fold and 8 -fold increased risk of dying from sarcomatoid carcinoma of the bladder, respectively [1, 3-8].

There is no standard treatment for sarcomatoid carcinoma due to the rareness and the lack of randomized controlled trials. No significant difference in overall survival was observed between patient populations treated with separate transurethral bladder tumor resection or radical cystectomy. Some investigators suggest neoadjuvant or adjuvant chemotherapy and/or radiotherapy. Outcomes associated with chemotherapy and radiotherapy are inconsistent in the large series. Recently has been suggested that early diagnosis and gemcitabine/cisplatin can positively affect survival. Multiinstitutional clinical trials are needed to establish protocols for the treatment of sarcomatoid carcinoma of the urinary bladder [1, 3-10].

\section{Conflict of Interest}

All authors declare have no conflict of interest.

\section{References}

1. Dahm P, Gschwend JE. Malignant non-urothelial neoplasms of the urinary bladder: a review. Eur Urol. 2003;44(6):672-681.

2. Cheng L, Zhang S, Alexander R, Maclennan GT, Hodges KB, Harrison BT, Lopez-Beltran A, et al. Sarcomatoid carcinoma of the urinary bladder: the final common pathway of urothelial carcinoma dedifferentiation. Am J Surg Pathol. 2011;35(5):e34-46.

3. Lopez-Beltran A, Cheng L. Histologic variants of urothelial carcinoma: differential diagnosis and clinical implications. Hum Pathol. 2006;37(11):1371-1388.

4. Black PC, Brown GA, Dinney CP. The impact of variant histology on the outcome of bladder cancer treated with curative intent. Urol Oncol. 2009;27(1):3-7.

5. Wright JL, Black PC, Brown GA, Porter MP, Kamat AM, Dinney CP, Lin DW. Differences in survival among patients with sarcomatoid carcinoma, carcinosarcoma and urothelial carcinoma of the bladder. J Urol. 2007; 178(6):2302-2306; discussion 2307.

6. Wang J, Wang FW, Lagrange CA, Hemstreet Iii GP, Kessinger A. Clinical features of sarcomatoid carcinoma (carcinosarcoma) of the urinary bladder: analysis of 221 cases. Sarcoma. 2010;2010.

7. Cheng L, Lopez-Beltran A, Bostwick DG. Sarcomatoid carcinoma (carcinosarcoma). In Bladder Pathology. Hoboken, New Jersey. Wiley-Blackwell. 2012;355-381.

8. Humphrey PA. Sarcomatoid carcinoma of the bladder. J Urol. 2013;189(4):1534-1535.

9. Willis DL, Porten SP, Kamat AM. Should histologic variants alter definitive treatment of bladder cancer? Curr Opin Urol. 2013;23(5):435-443.

10. Baseskioglu B, Duman BB, Kara IO, Can C, Yildirim M, Acikalin M. Early detection and gemcitabine/cisplatin combination positively effect survival in sarcomatoid carcinoma of the urinary bladder. Asian Pac J Cancer Prev. 2012;13(11):5729-5733. 